\title{
Intensive geriatric rehabilitation reduced hospital stay and time to independent living in hip fracture patients with mild to moderate dementia
}

\author{
Huusko TM, Karppi P,Avikainen Vet al. Randomised, clinically controlled trial of intensive geriatric rehabilitation in \\ patients with hip fracture: subgroup analysis of patients with dementia. BMJ 2000 Nov 4;321:1107-11.
QUESTION: In hip fracture patients with dementia, is intensive geriatric rehabilitation (IGR) effective and does its effectiveness vary with degree of dementia?

Design
Preplanned subgroup analysis of a randomised (unclear
allocation concealment), unblinded, controlled trial with 1 year of follow up.

Setting

Jyväskylä Central Hospital, Finland.

\section{Patients}

260 independently living patients $\geqslant 65$ years of age (mean age 80 y, $72 \%$ women) who were admitted to hospital with a hip fracture. Exclusion criteria were inability to walk unaided before the fracture, pathological fractures, multiple fractures, serious early complications, receiving calcitonin treatment, or terminal illness. 238 patients (92\%) completed the study.

\section{Intervention}

After surgery for hip fracture, 130 patients were allocated to IGR, which consisted of providing advice, training, drug treatment, physiotherapy, occupational therapy, speech therapy; listening to patients' concerns; and help with use of appliances, equipment, and daily living aids all provided within the geriatric ward of the Central Hospital. 130 patients were allocated to the control group, which involved discharge to a local hospital.

Sources of funding: Central Finland Healthcare District; Kuopio University Hospital; Emil Aaltonen Foundation; Uulo Arhio Foundation; Novartis

Finland.

For correspondence: Dr T M Huusko, Department of Public Health and General Practice, University of Kuopio, PO Box 1627 70211 Kuopio, Finland.

\section{Main outcome measures}

Length of hospital stay, independent living, and mortality 3 months and 1 year after surgery.

\section{Main results}

In patients with normal scores on the Mini Mental State Examination (MMSE) and in those with scores indicating severe dementia, no difference existed between the intervention and control groups for length of hospital stay. For patients with mild and moderate dementia, however, hospital stay was shorter for those in the intervention group (table 1). More patients with mild and moderate dementia

Table 1 Length of hospital stay (d) according to Mini Mental State Examination Score

\begin{tabular}{|c|c|c|c|c|c|}
\hline \multirow[b]{2}{*}{ Score } & \multicolumn{2}{|c|}{ Intervention group } & \multicolumn{2}{|c|}{ Control group } & \multirow[b]{2}{*}{ p Value } \\
\hline & $\mathbf{N}$ & Median stay (range) & $\mathbf{N}$ & Median stay (range) & \\
\hline 18-23 (mild dementia) & 35 & $29(16$ to 138$)$ & 42 & 46 (10 to 365$)$ & 0.002 \\
\hline 12-17 (moderate dementia) & 24 & 47 (10 to 365$)$ & 12 & 147 (18 to 365$)$ & 0.042 \\
\hline
\end{tabular}

Table 2 Intensive geriatric rehabilitation (IGR) v control procedure in patients with mild to moderate dementia

\begin{tabular}{llllll} 
Outcomes at 3 months & IGR & Control & RBI (95\% CI) & NNT (CI) \\
Independent living-mild dementia & $91 \%$ & $67 \%$ & $37 \%(9$ to 80$)$ & 5 (3 to 16$)$ \\
\hline Independent living-moderate dementia & $63 \%$ & $17 \%$ & $275 \%(28$ to 1267$)$ & 3 (2 to 9$)$ \\
\hline
\end{tabular}

Abbreviations defined in glossary; RBI, NNT, and Cl calculated from data in article. in the intervention group were living independently at 3 months (table 2); this difference was no longer statistically significant at 1 year. No difference existed between the groups for mortality.

\section{Conclusion}

In hip fracture patients with mild to moderate dementia, intensive geriatric rehabilitation led to fewer days in hospital and more patients were able to return to independent living.

\section{COMMENTARY}

This study by Huusko et al is the first randomised controlled trial to examine the effectiveness of IGR for hip fracture patients with varying levels of dementia. Length of hospital stay was dramatically shorter for patients with mild and moderate dementia who received the IGR. Although more patients with mild and moderate dementia in the intervention group were able to return to independent living by 3 months, the difference by 1 year was no longer significant; numbers in these subgroups, however, were fairly small.

The large sample of geriatric patients with hip fractures was selected from 1 hospital and it took $>4$ years to recruit the 260 patients. Although the large sample size is a strength, the time span may be a limitation as treatments, staff, and length of hospital stay could have changed over such a long period of time.

Another possible limitation is the assessment of cognitive impairment using only the MMSE. The MMSE is suitable as a screening instrument but not as a diagnostic tool, and therefore other tools should have been used before allocating patients to dementia groups. The MMSE was done only once, 10 days after surgery, and nothing is known about the patient's status on discharge.

Nevertheless, the trial is remarkable and the results are of interest to everyone who is involved in the treatment of these patients, as well as to geriatric rehabilitation in general. The geriatric team was multidisciplinary, including a geriatrician internist, a specially trained general practitioner, nurses with training in the care of older patients, a social worker, a neuropsychologist, an occupational therapist, physiotherapists, a consultant specialist in physical medicine, a neurologist, and a psychiatrist. As well as working with patients and families, the team also collaborated with community health services such as home care. This study is a good example of the value of multidisciplinary teams who bring together hospital and community services to meet the physical, social and psychological needs of patients and families. As our population ages, there will be increasing numbers of demented patients with hip fractures. Further research with larger sample sizes may be needed to evaluate the long term effects of this intervention. Also, an economic analysis is warranted.

Michael Schulz, RN PhD student, Martin Luther University Institute for Nursing and Health Science Halle/Saale, Germany 